\title{
A study of drug attitude and medication adherence and its relationship with the impact of illness among the mentally ill
}

\author{
DushaD RAM 1 \\ https://orcid.org/0000-0003-4908-5407
}

MiNTU MATHEW2 2

Samaksha Pasupalate Bheemaraju ${ }^{3}$

https://orcid.org/0000-0003-3183-4318

\section{Basavana Gowdappa Hathur ${ }^{4}$}

https://orcid.org/0000-0003-0789-7511

1 Department of Psychiatry, JSS Hospital, Mysore, karnataka, India.

2 Department of Clinical Pharmacy, JSS Hospital, Mysore, karnataka, India.

3 Department of Clinical Pharmacy, JSS Hospital, Mysore, karnataka, India.

${ }_{4}^{4}$ Department of Medicine, JSS Hospital, Mysore, karnataka, India.

Institution where the study was conducted: Department of Psychiatry, JSS Hospital, Mysore, karnataka, India.

Received: 2/13/2018- Accepted: 6/16/2019

DOI: 10.1590/0101-60830000000201

\begin{abstract}
Background: Impact of illness may vary with the medication adherence which in turn may vary with the attitude towards drugs. There is a paucity of research examining relationships between these variables. Objective: To study the levels of drug attitude, adherence and its relationship with the impact of illness. Methods: A total of 279 participants with mental illness in remission were assessed with socio-demographic and clinical proforma, scales like Hogan Drug Attitude Inventory (DAI), Impact of Illness Scale (IIS), and Morisky Medication Adherence Scale (MMAS) were used. Results: Mean score on DAI, IIS, and MMAS were 2.38 $(\mathrm{SD}=4.6), 25.88(\mathrm{SD}=6.6)$, and $5.04(\mathrm{SD}=2.2)$ respectively. On linear regression analysis $\left(\mathrm{R}_{2}=.122, \mathrm{DF}=2, \mathrm{~F}=17.598, \mathrm{p}<.001\right)$ IIS Score was statistically significant but negatively associated with the score of MMAS $(\mathrm{p}<.05)$ and DAI $(\mathrm{p}<.05)$. Discussion: Impact of illness has an inverse relationship with the level of drug attitude and medication adherence. Improving drug attitude and adherence may buffer the impact of illness.
\end{abstract}

Ram D et al. / Arch Clin Psychiatry. 2019;46(4):85-8

Keywords: Drug attitude, adherence, illness impact, mental illness.

\section{Introduction}

Mental illnesses often have direct or indirect consequences, leading to distress and disability that undermine the quality of life ${ }^{1}$. Impact of mental illness includes feeling restricted, disrupted personal and family routine, problems in personal, emotional \& social adjustment, financial strain, feeling overwhelmed and burdened ${ }^{2}$. It is determined by severity of the symptoms, duration of the episodes, forms of the symptoms, treatment response, coping skills, stress load, comorbidity, and lifestyle. However, it remains unclear as to which factors best predict the quality of life ${ }^{3}$. Being a leading cause of DALY, and a neglected area of concern by the government like in India, and with a strong recommendation from World Health Organization for further research, it is important to explore the impact of mental illness among Indian population. To the best of our knowledge, no studies have explored the impact of mental illness in Indian population using standardised assessment tools. Mental illness results in adverse consequences $^{1}$, hence there is a need to know the level of the impact of mental illness.

Exploring the patient's attitude towards medication is important as it helps to better understand the patients. Attitude of patients toward medication depends on multiple factors like overall perception of medications as good or bad, effect and side effects of the medication, and willingness to opt for medication ${ }^{4}$. More positive attitude towards medication and adherence has been associated with better insight (in the presence of a mental disorder) and sharing a good relationship with the treating physician. Predictors of positive attitude towards medication in mental illness includes insight into the need for treatment, a higher attribution of the symptoms to a disorder, experience of less negative side effects, presence of biological causal beliefs, and less endorsement of psychological causal beliefs ${ }^{5}$. A positive attitude towards medication has a positive correlation with adherence ${ }^{6,7}$. So far, no study has examined drug attitude among Indian population using standardised rating scale.

The WHO defines adherence in long-term therapy as "the extent to which a person's behaviour taking medication, following a diet, and/or executing lifestyle changes - corresponds with agreed recommendations from a health care provider"s WHO also reported that only $50 \%$ of patients with chronic diseases adhere to treatment recommendations in developed countries and further quoted that "increasing the effectiveness of adherence interventions may have a far greater impact on the health of the population than any improvement in specific medical treatments"8. Attitude towards medication may directly or indirectly mediate the impact of illness. A negative attitude, delay or refusal of treatment may lead to higher impact of illness. Indirect evidence suggests that better attitude towards medication may alleviate the impact of illness ${ }^{9}$.

Keeping in view that mental illness is not only associated with adverse consequences which vary with the levels of adherence, but drug attitude may also influence the adherence, hence it may indirectly determine the impact of illness. There is a knowledge gap as to what are the levels and relationships of these variables. As per our knowledge, no study has examined the relationship between these variables which further warrants a need to examine these variables.

With the above background, this study was conducted to know the drug attitude andmedication adherence and their relationship with impact of illness, among those with mental illnesses. We hypothesized that impact of illness has an inverse relation with level of drug attitude and adherence. 


\section{Methods}

The study was hospital based cross sectional study and was conducted at the Outpatient Department of Psychiatry, JSS medical college, Mysore, India. The subjects were recruited from March 2018 till September 2018. Two hundred and seventy nine participants, who were living in the community after an episode of mental illness and attended outpatient department of psychiatry during follow-up, were recruited after obtaining an informed consent. Patients who were between age of 18-65 years of age, with at least one episode of mental illness in the past but currently in remission (as ascertained by the treating psychiatrist through a detailed clinical examination) were included in the study. Those who had chronic physical illness or disability (as it may influence the perception of impact of mental illness) were excluded from the study. Participants with psychiatric diagnosis of Mental Retardation and Dementia were also excluded due to reliability issue. Participants who met the selection criteria underwent further assessment with the following tools:

1) Socio-demographic and clinical Proforma - which included age, gender, marital status, education, religion, occupation, domicile status, socio-economic status, medication preference, use of self-medication and diagnosis.

2) Drug Attitude Inventory (DAI) - To assess the attitude towards medication, DAI was used after translating it to Kannada language. This tool was initially developed in $1983^{2}$. This self-administered tool has 30 items with true/false response options. Each correct answer is rewarded with 1 point while for a wrong answer, 1 point is deducted. It has good internal consistency $(\alpha=0.88)$, test-retest reliability $(r=0.99, p<10)$ and inter-rater reliability $(\text { ICC }=0.99)^{4}$.

3) Impact of Illness Scale (IIS) - This scale was developed by Klimidis et al. in 2001, to assess impact of illness ${ }^{10}$. The IIS is a short, easy to administer scale, and has a high internal consistency coefficient alpha of 0.93 . It has been used in diverse socio-cultural settings to measure the illness impact. The scale assesses areas of relationship, work, joy \& recreation, social \& religious obligation, family obligation, routine chores, daily needs, mobility, and participation. Studies suggest that IIS is a reliable and gives valid measure of psychosocial impact of illness that may be applicable in a wide range of socio-cultural settings ${ }^{10}$. In our study this scale too was administered after translation to Kannada.

4) Morisky Medication Adherence Scale- This is a self-reporting scale and has 8 items with yes/no response options. Correct response is given 1 point, while incorrect response is scored 0 . Score of $<6$ indicates low adherence, score of 6-8 indicates medium adherence, and a score of $\geq 8$ indicates high adherence. This scale has a sensitivity and specificity of $93 \%$ and $53 \%$ respectively, and Cronbach's alpha value of $0.83{ }^{11}$. A translated Kannada version was used in this study.

The data was analyzed using SPSS version 21. Descriptive statistics were used to analyze demographic and clinical features. Exploratory analysis was done to the find out the relationships between DAI, MMAS and IIS score with socio-demographic and clinical variables, while linear regression analysis was done to know the relationship of score on DAI and MMAS with the score on IIS.

\section{Results}

In this study, the majority of subjects were educated, married, unemployed, from a rural background, Hindus, from low socioeconomic status, had preferences for modern treatment, had a history of self-medication in the past, and had a past episode of mood disorder (Table 1).

Mean age of participants was $38.42(\mathrm{SD}=12.6)$ years. Mean score on DAI, IIS, and MMAS were $2.38(\mathrm{SD}=4.6), 25.88$ (SD = $6.6)$, and $5.04(\mathrm{SD}=2.2)$ correspondingly (Table 2$)$. On the score of DAI, no statistically significant group difference was observed among socio-demographic variables except for domicile status (MU
$=5571.50, \mathrm{Z}=-1.86, \mathrm{p}<.05)$ and education $\left(\chi^{2}=6.771, \mathrm{DF}=2, \mathrm{p}\right.$ $<.05$ ) (Tables 3 and 4).

Similarly on the IIS score, no statistically significant group difference was observed among socio-demographic variables except occupation $(\mathrm{MU}=6.88, \mathrm{Z}=-2.02, \mathrm{p}<.05)$ and diagnosis $\left(\chi^{2}=8.92\right.$, $\mathrm{DF}=2, \mathrm{p}<.05)($ Table 3 and 4$)$.

On the score of MMAS, statistically significant group difference was observed for demographic variables - Gender $(\mathrm{MU}=7104.50$, $\mathrm{Z}=-2.23, \mathrm{p}<.05)$, Medication Preference $(\mathrm{MU}=1302.00, \mathrm{Z}=$ $-1.98, \mathrm{p}=<.05)$, and Self-Medication $\left(\chi^{2}=17.36, \mathrm{DF}=2, \mathrm{p}<.001\right)$ (Tables 3 and 4 ).

On linear regression analysis $\left(\mathrm{R}_{2}=.122, \mathrm{DF}=2, \mathrm{~F}=17.59, \mathrm{p}<\right.$ $.001)$ IIS Score was statistically significant but negatively associated with the score of MMAS $(\mathrm{p}<.05)$ and DAI $(\mathrm{p}<.05)($ Table 5).

Table 1. Socio-demographic and clinical characteristics

\begin{tabular}{|c|c|c|}
\hline Variables & $n$ & $\%$ \\
\hline \multicolumn{3}{|l|}{ Gender } \\
\hline Male & 144 & 49.8 \\
\hline Female & 145 & 50.2 \\
\hline \multicolumn{3}{|l|}{ Occupation } \\
\hline Employed & 128 & 44.3 \\
\hline Unemployed & 161 & 55.7 \\
\hline \multicolumn{3}{|l|}{ Education } \\
\hline Uneducated & 34 & 11.8 \\
\hline Primary & 57 & 19.7 \\
\hline Up to PUC & 173 & 59.9 \\
\hline Graduate & 25 & 8.7 \\
\hline \multicolumn{3}{|l|}{ Residence } \\
\hline Rural & 204 & 70.6 \\
\hline Urban & 85 & 29.4 \\
\hline \multicolumn{3}{|l|}{ Religion } \\
\hline Hindu & 267 & 92.4 \\
\hline Muslim & 19 & 6.6 \\
\hline Christian & 3 & 1.0 \\
\hline \multicolumn{3}{|c|}{ Socio-economic status } \\
\hline Low & 201 & 69.6 \\
\hline Middle & 83 & 28.7 \\
\hline High & 5 & 1.7 \\
\hline \multicolumn{3}{|c|}{ Marital status } \\
\hline Single & 69 & 23.9 \\
\hline Married & 220 & 76.1 \\
\hline \multicolumn{3}{|c|}{ Medication preference } \\
\hline Allopathic & 272 & 94.1 \\
\hline Ayurvedic & 17 & 5.9 \\
\hline \multicolumn{3}{|c|}{ Self-medication } \\
\hline Never & 84 & 29.1 \\
\hline Some time & 171 & 59.2 \\
\hline Frequently & 34 & 11.8 \\
\hline \multicolumn{3}{|l|}{ Diagnosis } \\
\hline $\mathrm{F} 10$ & 52 & 18.0 \\
\hline $\mathrm{F} 20$ & 14 & 4.8 \\
\hline F30 & 187 & 64.7 \\
\hline F40 & 36 & 12.5 \\
\hline
\end{tabular}

Table 2. Socio-demographic and clinical characteristics

\begin{tabular}{|l|c|c|c|c|}
\hline Variables & Minimum & Maximum & Mean & Std. deviation \\
\hline Age & 15 & 73 & 38.26 & 12.48 \\
\hline DAI Score & -10.00 & 10.00 & 2.38 & 4.46 \\
\hline IIS Score & .00 & 36.00 & 25.88 & 6.59 \\
\hline MMAS Score & .00 & 7.00 & 5.04 & 2.23 \\
\hline
\end{tabular}


Table 3. Level of group difference of socio-demographic variables on DAI, IIS and MMAS Score

\begin{tabular}{|c|c|c|c|c|c|}
\hline Variables & $\mathrm{N}$ & Mean Rank & $\mathrm{MU}$ & Z & $p$ \\
\hline \multicolumn{6}{|c|}{ Residence* DAI Score } \\
\hline Rural & 204 & 135.18 & 5571.50 & -1.864 & .050 \\
\hline Urban & 85 & 115.75 & & & \\
\hline \multicolumn{6}{|c|}{ Occupation *IIS Score } \\
\hline Employed & 128 & 139.83 & 6.88 & -2.029 & .042 \\
\hline Un-employed & 161 & 121.02 & & & \\
\hline \multicolumn{6}{|c|}{ Gender *MMAS Score } \\
\hline Male & 144 & 119.94 & 7104.50 & -2.239 & .025 \\
\hline Female & 145 & 18437.50 & & & \\
\hline
\end{tabular}

Table 4. Level of group difference of socio-demographic variables on DAI, IIS Score and MMAS Score

\begin{tabular}{|c|c|c|c|c|}
\hline Variables & $\mathrm{N}$ & Mean Rank & df & $p$ \\
\hline \multicolumn{5}{|c|}{ Education* DAI Score } \\
\hline Primary & 34 & 131.91 & 2 & .034 \\
\hline Up to PUC & 57 & 110.06 & & \\
\hline Up to graduate & 173 & 96.00 & & \\
\hline \multicolumn{5}{|c|}{ Diagnosis* IIS Score } \\
\hline F10 & 52 & 122.02 & 3 & .030 \\
\hline F20 & 14 & 156.62 & & \\
\hline F30 & 187 & 134.92 & & \\
\hline F40 & 36 & 97.44 & & \\
\hline \multicolumn{5}{|c|}{ Self-medication* MMAS Score } \\
\hline Never & 84 & 143.77 & 2 & .001 \\
\hline Some time & 171 & 132.85 & & \\
\hline Frequently & 34 & 83.68 & & \\
\hline
\end{tabular}

Table 5. Relationships of drug attitude and medication adherence with impact of illness

\begin{tabular}{|c|c|c|c|c|c|c|c|c|}
\hline \multirow{2}{*}{ Model } & & \multicolumn{2}{|c|}{ Unstandardized coefficients } & \multirow{2}{*}{$\begin{array}{c}\begin{array}{c}\text { Standardized } \\
\text { coefficients }\end{array} \\
\text { Beta }\end{array}$} & \multicolumn{2}{|c|}{$95 \%$ confidence interval for $B$} & \multirow{2}{*}{$\mathrm{t}$} & \multirow{2}{*}{ Sig. } \\
\hline & & B & Std. Error & & Lower bound & Upper bound & & \\
\hline \multirow[t]{3}{*}{1} & (Constant) & 10.200 & .408 & & 28.205 & 33.022 & 24.986 & .001 \\
\hline & DAI Score & -.082 & .034 & -.171 & -.436 & -.037 & -2.424 & .016 \\
\hline & MMAS Score & -.218 & .069 & -.223 & -1.062 & -.248 & -3.157 & .002 \\
\hline
\end{tabular}

R2: .122; df: 2, F: 17.598; p: .001; Dependent Variable: IIS Score; Predictors: MMAS Score, DAI Score.

\section{Discussion}

The demographic and clinical characteristics were similar to the previous studies conducted at the same centre ${ }^{12}$. The study centre is a charitable trust catering services to both rural and urban population who are mostly Hindu by religion, and under privileged. In our study, we observed a positive attitude towards drugs (mean of 2.38 out of maximum score of 10). The study centre offers evidence based modern treatment that is supposed to be effective. Consistent to our previous reports, in this study also, the majority of the participants (94\%) preferred modern treatment, compared to other methods such as Ayurvedic medications. This could also be partially due to the higher prevalence of medical model of illness among participants attending study centre.

High mean score on 25.88 (maximum possible score 27) indicates a high impact of illness on the participants. Mental illness is known to affect interpersonal relationships, working capacity, leisure and recreational activity, familial obligation etc. Mental illness is also known to affect the quality of life of the affected.

Consistent with previous reports, we observed low levels of medication adherence. Medication adherence is usually poor in subjects with mental illness and many etiological factors play a role such as patient related factors, medication related factors and illness related factors.
Similar to our findings, other reports revealed varying levels of drug attitude, depending upon occupation in patients with schizophrenia ${ }^{13}$. Employed status makes people to have a rational view about medication in order to be fit for occupation and free from illness. Those who were employed were more likely to have higher levels of education than those without employment. Similarly those with mood, anxiety and other non-psychotic disorders may had a favourable attitude towards medication than those with psychotic illness. Research has revealed a disparity between men and women in their medication adherence, as observed in this study; that may require more personalized drug selection and therapeutic management to improve the outcome of an illness ${ }^{14}$.

Most participants had a preference for modern, mainstream treatment. This was congruent with the fact that modern medication offers evidence based remedy to the patients; hence better adherence was observed in comparison to those that preferred other methods such as Ayurvedic. There are reports that concordance depends on the patient, illness and clinician factors and patient choice is the final common pathway that may be operative in a modern treatment method 15 .

We observed a better adherence of medication with lower level of self-medication. Such observation has been made among nonpsychiatric populations with less severe illnesses ${ }^{16}$. The commonest 
cause of self-medication is usually symptomatic improvement and is often the main reason for poor compliance ${ }^{16}$.

Consistent with our hypothesis, we observed an inverse relationship between IIS score with DAI \& MMAS score. Lower positive drug attitude is associated with higher impact of illness, has been reported earlier ${ }^{17}$. Observed inverse relationship in this study did not appear to be mediated through compliance and illness remedial behaviour. Negative drug attitude is known to be a predictor of poor compliance and may aggravate the persistence of symptoms, negative effect of illness and psychosocial dysfunction such as social isolation, loss of employment, and loss of role and status. The pharmacophobic (those with a negative drug attitude) are often introverted and more likely to be sensitive and relatively less judgmental as compared to the pharmacophilic ${ }^{18}$. On the other hand, a positive drug attitude favours greater shared decision making and self-efficacy that are helpful in reducing impact of illness ${ }^{19}$.

With the findings of this study, it can be concluded that negative attitude towards medication, low medication adherence and high level of impact of illness presents in mental illness in remission. Impact of illness has an inverse relationship with level of drug attitude and medication adherence. Improving drug attitude and adherence may buffer the impact of illness. The findings of this study should be interpreted with caution, as it was constrained by its cross sectional study design, lack of control group, lack of assessment of insight, and for not using structured instruments for the assessment of the diagnosis. Further research is needed for addressing the limitations of this study.

\section{Acknowledgment}

The authors would like to thank Yahosha, Shamaya, Hagai, Asther, Yasuas, Marias (Divine Retreat Centre, Chalakudy, Kerala, India), Ashish, Akash, and Mini for their moral support.

\section{References}

1. Evans S, Banerjee S, Leese M, Huxley P. The impact of mental illness on quality of life: A comparison of severe mental illness, common mental disorder and healthy population samples. Quality Life Res. 2007;16(1):17-29.

2. Stoeckel M, Weissbrod C. Growing up with an ill parent: An examination of family characteristics and parental illness features. Fam Syst Health. 2015;33(4):356-62.

3. Barnes AL, Murphy ME, Fowler CA, Rempfer MV. Health-Related Quality of Life and Overall Life Satisfaction in People with Serious Mental Illness. Schizophr Res Treat. 2012;2012:245103.
4. Hogan TP, Awad AG, Eastwood R. A self-report scale predictive of drug compliance in schizophrenics: reliability and discriminative validity. Psychol Med. 1983;13(1):177-83.

5. Wiesjahn M, Jung E, Lamster F, Rief W, Lincoln TM. Explaining Attitudes and Adherence to Antipsychotic Medication: The Development of a Process Model. Schizophr Res Treat 2014;2014:341545.

6. Yang J, Ko YH, Paik JW, Lee MS, Han C, Joe SH, et al. Symptom severity and attitudes toward medication: impacts on adherence in outpatients with schizophrenia. Schizophr Res. 2012;134(2-3):226-31.

7. Chandra IS, Kumar KL, Reddy MP, Reddy CMPK. Attitudes toward Medication and Reasons for Non-Compliance in Patients with Schizophrenia. Indian J Psychol Med. 2014;36(3):294-8.

8. Sabate E. Adherence to long-term therapies: evidence for action. Geneva: World Health Organization, 2003. Available from: http://www.who.int/ chronic_conditions/en/adherence_report.pdf. Accessed on: Oct. 8, 2017.

9. Mohamed S, Rosenheck R, He H, Yuping N. Insight and attitudes towards medication among in patients with chronic schizophrenia in the US and China. Soc Psychiatry Psychiatr Epidemiol. 2014;49(7):1063-70.

10. Klimidis S, Minas IH, Yamamoto K. Impact of Illness Scale: Reliability, Validity, and Cross Cultural Utility. Compr Psychiatry. 2001;42(5):416-23.

11. Morisky DE, Ang A, Krousel-Wood M, Ward HJ. Predictive validity of a medication adherence measure in an outpatient setting. J Clin Hypertension (Greenwich). 2008;10(5):348-54.

12. Ram D, Benny N, Gowdappa B H. Relationship between Depression Literacy and Medication Adherence in Patients with Depression. J Mood Disor. 2016;6(4):183-8.

13. Chandra IS, Kumar KL, Reddy MP, Reddy CP. Attitudes toward medication and reasons for non-compliance in patients with schizophrenia. Indian J Psychol Med. 2014;36(3):294-8.

14. Manteuffel M, William S, Chen W, Verbrugge RR, Pittman DG, Steinkellner A. Influence of patient sex and gender on medication use, adherence, and prescribing alignment with guideline. J Women Health. 2014;23(2):112-9.

15. Mitchell AJ. Adherence behavior with psychotropic medication is a form of self-medication. Med Hypothesis. 2007;68(1):12-21.

16. Haque BS, Alam NN, Mushroor S, Sultana N. Association between SelfMedication Behavior and Adherence among Medical Students in Dhaka. Asian J Pharmace. 2017;11(2 Suppl):347-52.

17. Brain C, Allerby K, Sameby B, Quinlan P, Joas E, Karilampi U, et al. Drug attitude and other predictors of medication adherence in schizophrenia: 12 months of electronic monitoring (MEMS $\left(^{*}\right)$ ) in the Swedish COASTstudy. Eur Neuropsychopharmacol. 2013;23(12):1754-62.

18. Hong S, Kang SY, Yoon JU, Kang U, Seong GJ, Kim CY. Drug Attitude and Adherence to AntiGlaucoma Medication. Yonsei Med J. 2010;51(2):261-9.

19. De las Cuevas C, Penate W. To what extent psychiatric patients feel involved in decision making about their mental health care? Relationships with socio-demographic, clinical, and psychological variables. Acta Neuropsychiatr. 2014;26(6):372-81. 